\title{
Fault detection and analysis of capacitive components of capacitive voltage transformer
}

\author{
XUAN Haizhen ${ }^{1, a^{*}}$, WEN Caiquan ${ }^{1, b}$, SONG Yongjia ${ }^{1}$ and WEI Xin ${ }^{1}$ \\ ${ }^{1}$ Wuzhou Bureau, CSG EHV Power Transimission Company, Wuzhou, China
}

\begin{abstract}
Capacitor voltage transformer (hereinafter referred to as CVT) with the growth of the capacitance of the operation period of aging, the phenomenon of breakdown, resulting in measurement, automation, protection and other equipment abnormalities. Under the influence of preventive test procedures, lack of accuracy, insufficient capacity, low test voltage, the preventive test project can not detect the initial failure of the capacitor and the breakdown of a few capacitor elements. The operating voltage as reference voltage, estimate feasibility analysis of CVT capacitor element of the state by the state of the secondary voltage, and through the field, find out more abnormal CVT can achieve CVT capacitor element of online monitoring function is proposed.
\end{abstract}

\section{Introduction}

Capacitor voltage transformer (CVT) is an electrical equipment composed of capacitor voltage divider and electromagnetic unit of medium voltage. It is characterized by simple structure and lower cost at higher voltage. At present, CVT is generally used in $500 \mathrm{kV}$ substations in China[1,2].

CVT monitors the operation of power grid. However, the breakdown of capacitors could lead to abnormal secondary voltage and inconsistency between the monitored voltage and the actual voltage. In extreme cases, it will affect the operation of secondary equipment featuring automation or protective functions.

Case 1: In August, 2015, there was a huge voltage deviation of up to $2.5-3 \mathrm{kV}$ between two buses at a $500 \mathrm{kV}$ substation, which directly affected the AVC regulation at the dispatching side. After the power failure test at \#2 bus, the voltage error of phase $\mathrm{A}$ and $\mathrm{C}$ was found to be up to $+0.94 \%$ and $+0.70 \%$, far beyond the range of $\pm 0.2 \%$. However, the dielectric loss and capacitance values were found normal in the pre-test[3].

Case 2: When a $500 \mathrm{kV}$ transmission line resumed power transmission, the voltage exceeded $550 \mathrm{kV}$, which caused failure of the automatic synchronization device. In the test of percent ratio error, it was found that phase A, B and $\mathrm{C}$ had an error of $+1.16 \%,+1.12 \%$ and $+1.76 \%$, far exceeding the tolerance value. However, the dielectric loss and capacitance values were found normal in the pretest[3].

The breakdown of capacitor will lead to large deviation of secondary voltage and inaccurate measurement, which will directly affect the delivery of electricity. Compared with traditional electromagnetic PT, CVT has poorer transient responses due to energy storage components such as capacitors and damping reactors.
Capacitor breakdown often takes place under lightning overvoltage and operation overvoltage. If the breakdown of CVT capacitor component occurs simultaneously with lightning overvoltage and operation overvoltage, it may cause the failure of distance protection [4].

\section{Analysis of Possible Causes}

The capacitor set of CVT is formed by 1 to 4 coupling capacitors and capacitor voltage dividers. Each coupling capacitor or capacitor voltage divider is equipped with dozens of capacitor component connected in series and sealed with diphenylethane. All capacitor components of high-voltage capacitor $\mathrm{C} 1$ and medium-voltage capacitor $\mathrm{C} 2$ are installed in 1-4 ceramic sleeves.

At present, polypropylene film and capacitor paper composite medium (also known as "film and paper composite medium") is widely used at home and abroad [5]. With the increase of service life or defects in packaging, capacitor components may be broken down under operation overvoltage and lightning overvoltage. The breakdown of the remaining components will be accelerated as a result of increase in the withstand voltage, as shown in Figure 1. However, the latest all-film dielectric would greatly improve the insulation property of capacitors and reduce carbon pollutants created by composite dielectric after the breakdown of the coupling capacitor[6].

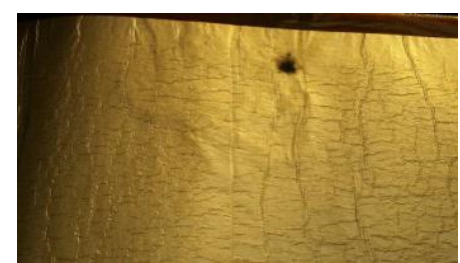

Figure 1a Initial stage of breakdown

\footnotetext{
"Corresponding author: ${ }^{\mathrm{a}} 18707749712 @ 163 . c 0 m$

bcsgwcq@139.com
}

(c) The Authors, published by EDP Sciences. This is an open access article distributed under the terms of the Creative Commons Attribution License 4.0 


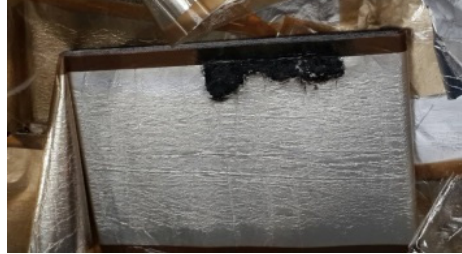

Figure 1b Complete breakdown

\section{Analysis of Capacitor Breakdown in Pre-test}

The Pre-test Regulation of Power Equipment requires CVT to go through high voltage test in case of power failure to ensure the capacitance value and dielectric loss test meet the requirement in such situation. The pre-test stipulates that the capacitance value of each section should be kept within the range of $-5 \% \sim+10 \%$ of rated value, and the tan $\delta$ value at $10 \mathrm{kV}$ shall not exceed $0.4 \%$ (film-paper composite insulation).

However, in the actual test, all the pre-test items of several abnormal CVT were qualified. The paper explained why the pre-test items did not accurately indicate the breakdown of capacitor components in the next section.

\subsection{Low standard set by the pre-test}

Assume each capacitor of C11, C12, C13 and C14 (including C2) of a CVT consists of 82 capacitor components in series, where the $\mathrm{C} 2$ capacitor is connected by 15 capacitor components in series. When there are a total of $\mathrm{n}$ capacitor components suffering from breakdown in any section of $\mathrm{C} 11, \mathrm{C} 12, \mathrm{C} 13$ and $\mathrm{C} 14$ (not including $\mathrm{C} 2)$, the capacitance deviation $\mathrm{P}=\mathrm{n} /(82-\mathrm{n}) * 100 \%$. When there is only one capacitor component of breakdown, the capacitance deviation is $+1.23 \%$. When there are two components of breakdown, the number goes up to $+2.50 \%$, all within the range. Therefore, when there is only one capacitor component experiencing breakdown, there is no need to handle it according to the pre-test procedure. In other words, when there is only a limited number of capacitor breakdown, it will not be considered as abnormal, and the CVT will have a percent ratio error way beyond tolerance.

\subsection{Relatively low voltage in the pre-test}

The large capacitance of CVT would cause insufficient capacity of field test instrument. According to the regulations, the applied voltage of each section was set at $10 \mathrm{kV}$. Given each section had 82 capacitor components, the applied voltage of each capacitor component was calculated at Usingle $=10000 / 82=122(\mathrm{~V})$, while the voltage after operation was $500 / \sqrt{ } 3 \mathrm{kV}$. Therefore, the applied voltage of each capacitor component was Usingle' $=500000 / \sqrt{ } 3 /(82 * 4)=880(\mathrm{~V})$. The voltage after operation was far higher than the test voltage.

In order to verify the influence of the test voltage on the test results, the CVT was returned to the factory for test, where the dielectric loss and capacitance values of
CVT were tested at $10 \mathrm{kV}$ and $72 \mathrm{kV}$ voltages respectively. The test results are shown in Table 1.

Table 1 Test results of CVT under different voltages

\begin{tabular}{ccccc}
\hline $\begin{array}{c}\text { Original } \\
\text { capacitanc } \\
\text { e value } \\
/ \mathrm{nF}\end{array}$ & $\begin{array}{c}\text { Tested at } \\
10 \mathrm{kV} \\
\text { voltages } \\
\operatorname{tg} \delta / \%\end{array}$ & $\begin{array}{c}\text { Tested at } \\
10 \mathrm{kV} \\
\text { voltages } \\
\mathrm{C}_{X} / \mathrm{nF}\end{array}$ & $\begin{array}{c}\text { Tested at } \\
72 \mathrm{kV} \\
\text { voltages } \\
\operatorname{tg} \delta / \%\end{array}$ & $\begin{array}{c}\text { Tested at } \\
72 \mathrm{kV} \\
\text { voltages } \\
\mathrm{C}_{X} / \mathrm{nF}\end{array}$ \\
\hline 20497 & 0.302 & $\mathbf{2 0 7 6 3}$ & 0.123 & $\mathbf{2 0 7 4 7}$ \\
20540 & 0.150 & 20540 & 0.118 & $\mathbf{2 0 8 0 1}$ \\
20317 & 0.161 & 20381 & 0.111 & 20382 \\
20428 & 0.303 & 20436 & 0.137 & $\mathbf{2 0 6 8 8}$ \\
\hline
\end{tabular}

It can be seen that the test voltage has a great impact on the test results. However, due to limitations of the field, it was impossible to further increase test voltage at the site. Meanwhile, it also shows that there may be two possible causes of capacitor breakdown. It could be caused by the direct adhesion of two polar plates, where the capacitance value of the unit was 0 under test voltage and operating voltage. It could also be caused by particles formed by dielectric breakdown and carbonization between electrode plates of capacitor component. The capacitance value was normal at $10 \mathrm{kV}$, while the capacitance value of the unit was 0 when the carbonized particles were directly conducted at high voltage in normal operation.

\subsection{Analysis of percent ratio error}

In addition to dielectric loss, capacitor breakdown was found in the test of percent ratio error. However, there are problems including:

1) Capacitors found to be unqualified in the test of percent ratio error was only labeled as abnormal according to the defect grading management, without sufficient attention to this problem. The cause of percent ratio error needed further test and analysis.

2) It is difficult to carry out the test of percent ratio error as few institutions have qualifications, and there are tough requirements of test instruments.

3 ) The test of percent ratio error has a long duration. As the Pre-test Regulation of Power Equipment has no requirement on percent ratio error of CVT, when the capacitance value of CVD and the factory value have a difference of over $2 \%$, or when the difference of the voltage division ratio between CVD and the factory value exceeds $2 \%$, transformers graded 0.5 and 0.2 in accuracy shall be tested. The Metrology Law of The People's Republic of China-Power Transformer requires the potential transformer to be tested every four years for error analysis.

4) There is no need to test the percent ratio error of CVT without metering equipment.

\section{Feasibility analysis based on secondary voltage monitoring}

The low voltage applied in the pre-test, the low requirements set by the pre-test procedures and the low 
precision of the dielectric loss tester make it difficult to determine the exact number of capacitor breakdown when there is only a limited number of capacitors involved in this situation. However, the data collected by secondary equipment during operation could be used for in-depth analysis to improve accuracy of measurement due to its high operating voltage and the sampling precision. Realtime test is required to examine CVT capacitor component. The principle goes as follows.

CVT is mainly composed of capacitive voltage divider and electromagnetic unit[7,8] (see Figure 2). The capacitive voltage divider includes two parts: main capacitor $\mathrm{C} 1$, voltage divider capacitor $\mathrm{C} 2$, as well as 1 to 4 porcelain sleeves outside. The electromagnetic unit is composed of intermediate transformer (i.e. intermediate voltage transformer $\mathrm{TV}$ ), compensation reactor $\mathrm{L}$ and damper, all of which are assembled in the same oil tank ${ }^{[7]}$ ${ }^{[8]}$. The structure diagram of CVT is shown in Figure 2:

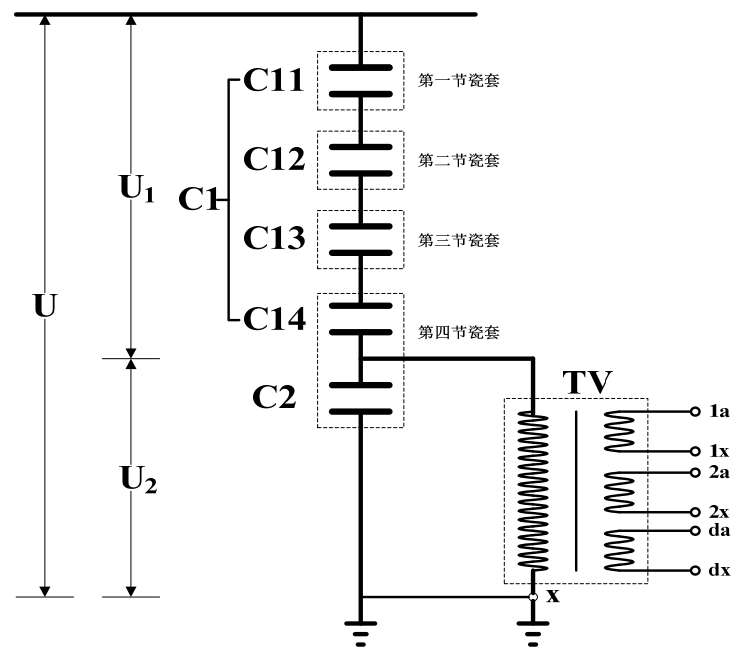

Figure 2 structure diagram of CVT

Assume that $\mathrm{C} 1$ in Figure 3 is formed by a total of $\mathrm{N}_{1}$ capacitors in series, $\mathrm{C} 2$ by a total of $\mathrm{N}_{2}$ capacitors in series, and the total number of capacitors is $\mathrm{N}$, where $\mathrm{N}=\mathrm{N}_{1}+\mathrm{N}_{2}$, then:

$$
U_{2}=U * \frac{N_{2}}{N}
$$

When there is one capacitor breakdown in $\mathrm{C} 1$ in Figure 2 ,

$$
U_{2}^{\prime}=U * \frac{N_{2}}{N-1}
$$

The ratio difference $\mathrm{f}$ of $\mathrm{CVT}$ at this time is

$$
f=\frac{U_{2}^{\prime}-U_{2}}{U_{2}} * 100 \%=\frac{1}{N-1} * 100 \%
$$

Similarly, when there are $\mathrm{n}$ capacitor component breakdown in $\mathrm{C} 1$, the ratio difference is $f=n /(N-n) * 100 \%$, where $\mathrm{N}$ is a constant. Therefore, the secondary value of voltage error $f$ (positive error) increases with the number of breakdown of capacitor component $n$.

Take a $500 \mathrm{kV} \# 2$ bus CVT in a substation as an example. There are four sections in the substation, including $\mathrm{C} 11, \mathrm{C} 12, \mathrm{C} 13$ and $\mathrm{C} 14$, each section with 82 capacitor components. $\mathrm{C} 2$ has 15 capacitor components.
And the total number of capacitors equals $\mathrm{N}=4 * 82=328$. According to the above formula of ratio difference, $\mathrm{f}_{1}=0.30 \%$ in the presence of breakdown of one capacitor component in $\mathrm{C} 1$, and $\mathrm{f}_{2}=0.60 \%$ when there are two capacitor components suffering from breakdown. However, the sampling precision of the measurement and control device can reach up to $0.2 \%$. Therefore, even if one capacitor breaks down, the sampling voltage of the measurement and control device can still obtain accurate results.

Therefore, to monitor the capacitor components of CVT, the zero-sequence voltage of CVT can serve as a reference, which is followed by the measurement and control device. When the zero-sequence voltage causes the breakdown of a single capacitor component, the CVT capacitor breakdown can be determined after excluding system voltage abnormality. Alternatively, it is plausible to compare its phase voltage with the accurate voltage of the system. When the error exceeds the set value, the CVT capacitor breakdown can be determined.

Through actual measurement, a number of CVT abnormalities have been found in a substation in more than one year. All the pre-test items of CVT were qualified, while they were found with breakdown after being returned to the factory for inspection. Therefore, this method can effectively evaluate capacitor components of CVT.

\section{Conclusion}

When there are only a limited number of capacitor breakdown in CVT, its capacitance value and dielectric loss remain within the tolerance range according to the pre-test regulations of South Power Grid. However, even if there is only one capacitor breakdown, the ratio difference is out of tolerance, which does not meet the requirement of precision and affects the accuracy of measurement. The breakdown has no influence on the operation of equipment, but could cause huge deviation of measurement. Therefore, there is inconsistency between the pre pre-test procedures and the requirement of precision.

It is difficult to determine the breakdown of capacitor in CVT under normal operation, as it is difficult to find large deviation of secondary voltage without a standard value for reference. The monitoring system could follow the change of zero-sequence voltage in real-time scenario. Therefore, changes of the zero-sequence voltage, combined with the actual system voltage in real time, could be used to determine the presence of internal faults in CVT.

Large deviation of secondary voltage of CVT is categorized as other types of defects of equipment by the South Power Grid, which is equivalent to equipment abnormality. However, such defects are not graded in the metering or measuring system, that is, there is no need to take care of it. When the secondary voltage abnormality affects the accuracy of measurement, no relevant measures are available to deal with the abnormality of CVT.

In case of rises in the secondary voltage during operation, if the capacitance value and dielectric loss are 
found to be qualified in the pre-test, it is recommended to use a high voltage dielectric loss meter for the experiment.

\section{References}

1. He, J., Yu, R., Yang, Y.J. The Common on Faults and monitoring of CVT. J. Power Capacitor Reactive Power Compensation, 30(5): 38-42 (2009).

2. Wu, D.W., Zhou, H.G. Analysis of Breakdown in capacitor voltage divider Component of 500kV CVT. J. Power Capacitor Reactive Power Compensation, 32(6):74-76,79 (2011).

3. Q/CSG114002-2011,Preventive test procedure for power equipment of China Southern Power Grid Co., Ltd

4. Shi, J.H., Suo, N.J.L., Xu, Q.Q., Liu, J.J., Ge, Y.Z. Digital Simulations on Transient Response Characteristics of capacitor voltage transformer and its Influence on distance Relays. J. Journal of Xian Jiaotong University, 37(4):415-419 (2003).

5. Fang, J.L. Research on the Technology Development of capacitor voltage transformer. J. Power Capacitor Reactive Power Compensation, (2) (2007).

6. Wang, R.D. The Effect on CVT Dielectric Loss Test of Various Test Method. D. Shandong University of technology (2008).

7. Guo, L.J., Xu, Y.J. Reason Analysis and Treatment of Over standard Dielectric Loss of $500 \mathrm{kV}$ Capacitor Voltage Transformer. J. Guangxi Electric Power, 32(1) (2009).

8. Ding, T., Chen, Z.Y., Liu, Z., Gao, L.M. Analysis and treatment of voltage abnormality in a $500 \mathrm{kV}$ Capacitor Voltage Transformer. J. Power Capacitor Reactive Power Compensation, 31(2): 52-55 (2010). 